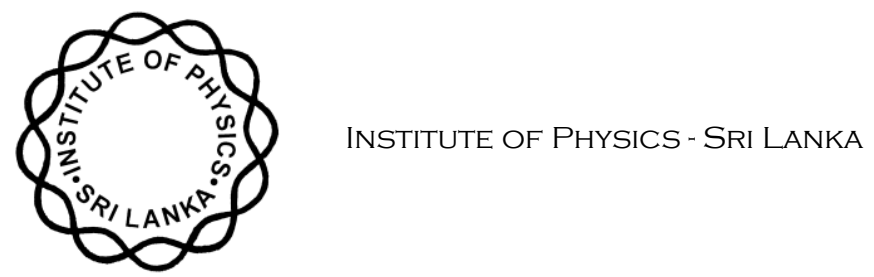

Research Article

\title{
Simultaneous analysis of non-strange negative parity baryon Properties in large $\mathbf{N}_{c} \mathbf{Q C D}$
}

\author{
Shavindra P. Premaratne, C.P. Jayalath ${ }^{\mathbf{1}}$ \\ Department of Physics, Faculty of Science, University of Peradeniya, Sri Lanka
}

\begin{abstract}
Non-strange $l=1$ excited baryons are analyzed within the Large $N_{c}$ QCD framework. The Large $N_{c}$ approach is used as an effective theory to simultaneously fit the masses and decays with corrections up to $O\left(1 / N_{c}\right)$. Values of the mixing angles for the $S=1 / 2$ and $S=3 / 2$ nucleon mass eigenstates are obtained within the simultaneous analysis. The observed effective operator hierarchy is consistent with the previous studies conducted in this sector and improved results are obtained.
\end{abstract}

Keywords: Large $\mathrm{N}_{c}$, negative parity baryons

\section{INTRODUCTION}

Quantum Chromodynamics (QCD), the theory of strong interactions, is a strongly coupled theory at low and intermediate energies, which does not offer a small expansion parameter. QCD has a "running" coupling constant, $\alpha_{s}$, which depends on the energy of the particles being considered. Thus, perturbation theory, which had immense success in Quantum Electrodynamics (QED) due to the smallness of the fine structure constant $\alpha(\approx 1 / 137)$, fails in the low energy regime of $\mathrm{QCD}^{1}$. As the naturally occurring hadrons belong to this energy limit, non-perturbative approaches were developed to study their properties. Various models such as the constituent quark model $^{2}$, the bag model ${ }^{3}$, and the Skyrme model ${ }^{4}$ were employed to qualitatively describe the baryon properties. However, the results obtained were dependent on the model used ${ }^{5}$. At present, Lattice QCD is the most widely used non-perturbative technique to study hadron properties.

\footnotetext{
${ }^{1}$ Corresponding author Email : jacpjayalath@gmail.com
} 
Hooft $^{6}$ proposed to generalize the number of colors to an arbitrary number, $N_{c}$, and paved the way for a method to study hadron properties as an alternative to methods such as Lattice QCD. The expansion parameter used in 't Hooft's model was $1 / N_{c}$, and the perturbative techniques in terms of $1 / N_{c}$ could be applied both in low and high energy regimes of QCD. The theory simplified in the $N_{c} \rightarrow \infty$ limit. Witten first applied this method to study baryons ${ }^{7}$ and formulated the large $N_{c}$ counting rules. Witten also proved that large $N_{c}$ baryons should be composed of $N_{c}$ valence quarks and then the baryon mass should be of $O\left(N_{c}\right)$.

Baryon spin-flavor symmetry at large $N_{c}$ was discovered by Gervais \& Sakita ${ }^{8,9}$ in 1984 and independently by Dashen \& Manohar ${ }^{10}$ in 1993. This has enabled the use of the $1 / N_{c}$ expansion in baryon phenomenology. In the QCD baryon sector, it was discovered that an exact contracted spin-flavor symmetry $S U\left(2 N_{f}\right)$ exists for large $N_{c}$, where $N_{f}$ is the number of light quark flavors ${ }^{8,9,10}$. This symmetry has paved the way for an expansion in $1 / N_{c}$ of QCD operators to be developed for baryon properties in terms of spin-flavor $S U\left(2 N_{f}\right)$ generators ${ }^{11}$. In operator analysis, effective operator bases are constructed and ordered in powers of $1 / N_{c}$ with the ultimate objective of analyzing baryon observables. Hence, the $1 / N_{c}$ expansion is used as an effective theory with a base of QCD operators.

In the excited baryon sector, there have not been any well-established modelindependent methods for analysis of baryon properties. The constituent quark model is a good qualitative theory in this domain. The $1 / N_{c}$ approach has been applied to many aspects of excited baryons with a high degree of success. The symmetry for the excited baryons is $O(3) \times S U\left(2 N_{f}\right)$ where $O(3)$ corresponds to the spatial rotations and $S U\left(2 N_{f}\right)$ is the spin-flavor symmetry. In these resonance studies, the baryons are classified into groups as $\left[X, \ell^{P}\right]$ where $X$ is the dimension of the irreducible representation in $S U(6), \ell$ is the orbital excitation level and $P$ is the parity of the multiplet. In the current study, the non-strange members of the $\left[70,1^{-}\right]$mixed-symmetric baryon multiplet are analyzed. It should be noted that although there are 70 states possible according to the group theoretic analysis, a sizeable portion of them are spurious states. $\left[70,1^{-}\right]$is the lowest lying negative-parity excited baryon multiplet and one of the best explored. As this study is concerned with the non-strange members of the multiplet, only seven members are considered in the analysis.

There are two sets of $N^{*}$ states which have identical spin and isospin. Hence these states tend to mix, giving the following physical states ${ }^{12}$,

$$
\left(\begin{array}{l}
N_{J}^{*} \\
N_{J}^{*^{\prime}}
\end{array}\right)=\left(\begin{array}{cc}
\cos \theta_{2 J} & \sin \theta_{2 J} \\
-\sin \theta_{2 J} & \cos \theta_{2 J}
\end{array}\right)\left(\begin{array}{c}
{ }^{2} N_{J}^{*} \\
{ }^{4} N_{J}^{*}
\end{array}\right)
$$

where $J=1 / 2$ and $J=3 / 2$ is the total spin, $N_{J}^{*()}$ represent mass eigenstates and the notation ${ }^{2 S+1} N_{J}^{*}$ has been used with $S=1 / 2$ and $S=3 / 2$ for the quark spin contribution. 
The organization of the paper is as follows. In section 2, the technique used for the simultaneous analysis is described. The operator bases used and the corresponding matrix elements are given. Section 3 presents the detailed results along with graphical representations. The discussion of the results and the conclusion are given in section 4 .

\section{ANALYSIS TECHNIQUE}

\subsection{Background}

In this work, the $1 / N_{c}$ expansion is utilized as an effective theory and a basis of effective operators is extracted from previous independent studies of masses and nonstrange strong decays of the baryons concerned ${ }^{12,13,14}$. A suitable subset of effective operators is chosen based on the relative contribution and the order in $1 / N_{c}$. These operators are simultaneously fit with the available data to obtain a consistent value for the mixing angles of the $S=1 / 2$ and $S=3 / 2$ nucleon mass eigenstates. Data points with very high errors are removed in favor of obtaining a more reliable fit. Partial decay widths for three unobserved channels are predicted which are consistent with and improve upon the results of previous studies conducted ${ }^{12}$. The simultaneous fit for the masses and decays is conducted with corrections up to $O\left(1 / N_{c}\right)$.

The mixing angles are theoretically obtained using the mass matrices of $S=1 / 2$ and $S=3 / 2$ nucleons. The theoretical expressions for the angles are used as input parameters for the mixing present in strong decay amplitudes. Then the best fit for the analysis is obtained by minimizing the chi-squared value,

$$
\chi_{d o f}^{2}=\frac{1}{\left(v_{o b}-v_{p}\right)} \sum_{i}\left(\frac{\xi_{i}^{(t h)}-\xi_{i}^{(e m p)}}{\sigma_{i}^{(e m p)}}\right)^{2}
$$

where $i$ is an index running over all $v_{o b}$ empirically known observables considered, $v_{p}$ is the total number of independent parameters in the fit, $\xi_{i}^{(t h)}$ is the theoretical expression for the observable, $\xi_{i}^{(e m p)}$ is the empirical value and $\sigma_{i}^{(e m p)}$ is the error associated with it. The above value is minimized using the coefficients of the effective operators. The degrees of freedom, $v_{d o f}$ is determined as

$$
v_{d o f}=v_{o b}-v_{p}
$$

\subsection{Operator Basis for Mass Analysis}

In the studies by Carlson et al. ${ }^{13,14}$, nine operators are obtained with corrections up to $O\left(1 / N_{c}\right)$. However, their analysis reveals that the first six operators are the most significant contributors to the baryon masses. Hence, in this study, only the first six operators are considered for the analysis. 
The matrix elements of the operators used in the baryon mass analysis are shown in Table 1. The third and sixth rows correspond to off-diagonal matrix elements. These operators were used to construct the theoretical expressions for the masses of seven baryons as,

$$
M^{*}=\sum_{i} c_{i} O_{i}
$$

where $c_{i}$ are the dynamical coefficients which have to be determined by fitting to experimental data given by $\mathrm{PDG}^{15}$.

To obtain the mixing angles, first the mass matrices are formed as,

$$
\mathrm{M}=\left(\begin{array}{cc}
N_{J}^{*} & N_{J}^{*^{\prime}}-N_{J}^{*} \\
N_{J}^{*^{\prime}}-N_{J}^{*} & N_{J}^{*^{\prime}}
\end{array}\right)
$$

where $J=1 / 2$ and $J=3 / 2$. Then eigenvalues of above matrix are obtained and the corresponding eigenvalues are fit with the experimental masses. The mixing angles can then be obtained by calculating the eigenvectors.

\subsection{Operator Basis for Decay Analysis}

In the study by Goity et al. ${ }^{12}, 17$ effective operators are obtained for strong decays up to $O\left(1 / N_{c}\right)$. According to the quark model, the $\eta$ particle is a mixture of $u, d, s$ quarks and $\bar{u}, \bar{d}, \bar{s}$ antiquarks. As this study is concerned strictly with the nonstrange decays, any involving the $\eta$ particle were not included in the analysis. Hence, considering purely non-strange decays, 12 operators are at disposal for the analysis. All decays containing the emission of a $\pi$ meson, except $N(1535) \rightarrow \Delta \pi$ D-wave decay (due to unreliability) were used in the fit. The independent operators used are shown in Table 2. The ordering in $1 / N_{c}$ for the operators was performed following Goity et al. ${ }^{12}$ by studying the matrix elements and not necessarily using the bodyness of the operators. 
Table 1: Matrix elements $\left\langle O_{i}\right\rangle_{j}$ of 6 operators, that are linearly independent for $N_{c}=3^{13,14}$

\begin{tabular}{|c|c|c|c|c|c|c|}
\hline & $\left\langle O_{1}\right\rangle$ & $\left\langle O_{2}\right\rangle$ & $\left\langle O_{3}\right\rangle$ & $\left\langle O_{4}\right\rangle$ & $\left\langle O_{5}\right\rangle$ & $\left\langle O_{6}\right\rangle$ \\
\hline & $N_{c}\langle\mathrm{I}\rangle$ & $\langle\ell s\rangle$ & $\frac{1}{N_{c}}\left\langle\ell^{(2)} g G_{c}\right\rangle$ & $\left\langle\ell s+\frac{4}{N_{c}+1} \ell t G_{c}\right\rangle$ & $\frac{1}{N_{c}}\left\langle\ell S_{c}\right\rangle$ & $\frac{1}{N_{c}}\left\langle S_{c}^{2}\right\rangle$ \\
\hline$N_{1 / 2}^{*}$ & +1 & $+\frac{2\left(2 N_{c}-3\right)}{N_{c}}$ & 0 & -4 & $-\frac{2\left(N_{c}+3\right)}{N_{c}}$ & $+\frac{\left(N_{c}+3\right)}{N_{c}}$ \\
\hline$N_{1 / 2}^{*^{\prime}}$ & +1 & +5 & $+\frac{10\left(N_{c}+1\right)}{N_{c}}$ & 0 & -10 & +4 \\
\hline$N_{1 / 2}^{*^{\prime}}-N_{1 / 2}^{*}$ & 0 & $\begin{array}{c}+2 \sqrt{\frac{\left(N_{c}+3\right)}{2 N_{c}}} \\
\left(2 N_{c}-3\right)\end{array}$ & $+\frac{10\left(2 N_{c}-1\right)}{N_{c}} \sqrt{\frac{\left(N_{c}+3\right)}{2 N_{c}}}$ & $+2 \sqrt{\frac{\left(N_{c}+3\right)}{2 N_{c}}}$ & $\begin{array}{c}+2 \sqrt{\frac{\left(N_{c}+3\right)}{2 N_{c}}} \\
\left(N_{c}+3\right)\end{array}$ & $\begin{array}{c}0 \\
\left(N_{c}+3\right)\end{array}$ \\
\hline$N_{3 / 2}^{*}$ & +1 & $-\frac{N_{c}}{N_{c}}$ & 0 & +2 & $+\frac{c}{N_{c}}$ & $+\frac{c}{N_{c}}$ \\
\hline$N_{3 / 2}^{*^{\prime}}$ & +1 & +2 & $-\frac{8\left(N_{c}+1\right)}{N_{c}}$ & 0 & -4 & +4 \\
\hline$N_{3 / 2}^{*^{\prime}}-N_{3 / 2}^{*}$ & 0 & $+\sqrt{\frac{5\left(N_{c}+3\right)}{N_{c}}}$ & $-\frac{\left(2 N_{c}-1\right)}{N_{c}} \sqrt{\frac{5\left(N_{c}+3\right)}{N_{c}}}$ & $+\sqrt{\frac{5\left(N_{c}+3\right)}{N_{c}}}$ & $+\sqrt{\frac{5\left(N_{c}+3\right)}{N_{c}}}$ & 0 \\
\hline$N_{5 / 2}^{*}$ & +1 & -3 & $+\frac{2\left(N_{c}+1\right)}{N_{c}}$ & 0 & +6 & +4 \\
\hline$\Delta_{1 / 2}^{*}$ & +1 & -2 & 0 & 0 & -8 & +4 \\
\hline$\Delta_{3 / 2}^{*}$ & +1 & +1 & 0 & 0 & +4 & +4 \\
\hline \multirow{2}{*}{$\begin{array}{c}\text { Operator } \\
\text { Overall Factor }\end{array}$} & \multirow{2}{*}{$+N_{c}$} & 1 & 1 & 1 & 1 & 1 \\
\hline & & 6 & $\overline{96}$ & $2\left(N_{c}+1\right)$ & $6 N_{c}$ & $2 N_{c}$ \\
\hline
\end{tabular}


Table 2: Basis operators for the independent decay analysis ${ }^{12}$

\begin{tabular}{|c|c|c|c|c|}
\hline & Name & Operator & Bodyness & Order in $1 / N_{c}$ \\
\hline \multirow{4}{*}{$\sum_{i=1}^{\infty}$} & $B_{1}^{[0,1]}$ & $(\xi g)^{[0,1]}$ & 1B & 0 \\
\hline & $B_{2}^{[0,1]}$ & $\frac{1}{N_{c}}\left(\xi\left(s T_{c}\right)^{[1,1]}\right)_{[0, a]}^{[0,1]}$ & \multirow{3}{*}{$2 \mathrm{~B}$} & 1 \\
\hline & $B_{3}^{[0,1]}$ & $\frac{1}{N_{c}}\left(\xi\left(t S_{c}\right)^{[1,1]}\right)_{[0, a]}^{[0,1]}$ & & 1 \\
\hline & $B_{4}^{[0,1]}$ & $\frac{1}{N_{c}}\left(\xi\left(g S_{c}\right)^{[1,1]}\right)_{[0, a]}^{[0,1]}$ & & 1 \\
\hline \multirow{8}{*}{ 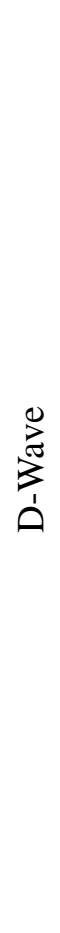 } & $B_{1}^{[2,1]}$ & $(\xi g)_{[i, a]}^{[2,1]}$ & 1B & 0 \\
\hline & $B_{2}^{[2,1]}$ & $\frac{1}{N_{c}}\left(\xi\left(s T_{c}\right)^{[1,1]}\right)_{[i, a]}^{[2,1]}$ & \multirow{5}{*}{$2 \mathrm{~B}$} & 1 \\
\hline & $B_{3}^{[2,1]}$ & $\frac{1}{N_{c}}\left(\xi\left(t S_{c}\right)^{[1,1]}\right)_{[i, a]}^{[2,1]}$ & & 1 \\
\hline & $B_{4}^{[2,1]}$ & $\frac{1}{N_{c}}\left(\xi\left(g S_{c}\right)^{[1,1]}\right)_{[i, a]}^{[2,1]}$ & & 1 \\
\hline & $B_{5}^{[2,1]}$ & $\frac{1}{N_{c}}\left(\xi\left(g S_{c}\right)^{[2,1]}\right)_{[i, a]}^{[2,1]}$ & & 1 \\
\hline & $B_{6}^{[2,1]}$ & $\frac{1}{N_{c}}\left(\xi\left(s G_{c}\right)^{[2,1]}\right)_{[i, a]}^{[2,1]}$ & & 0 \\
\hline & $B_{7}^{[2,1]}$ & $\frac{1}{N_{c}^{2}}\left(\xi\left(s\left(\left\{S_{c}, G_{c}\right\}\right)^{[2,1]}\right)^{[2,1]}\right)_{[i, a]}^{[2,1]}$ & \multirow{2}{*}{ 3B } & 1 \\
\hline & $B_{8}^{[2,1]}$ & $\frac{1}{N_{c}^{2}}\left(\xi\left(s\left(\left\{S_{c}, G_{c}\right\}\right)^{[2,1]}\right)^{[3,1]}\right)_{[i, a]}^{[2,1]}$ & & 1 \\
\hline
\end{tabular}

The notation used for the operators, $B_{\left[m_{P, I P 3}\right]}^{\left[l_{P}, I_{P}\right]}$, indicates the spin $l_{P}$ and isospin $I_{P}$ of the operator along with the respective projections $m_{P}$ and $I_{P 3}$. For simplicity, the spin and isospin projections have been omitted in some operators. The final set of independent operators has been constructed by coupling the orbital transition operator, $\xi^{[1,0]}$, with the spin-flavor operators and calculating the matrix elements explicitly ${ }^{12}$. The decay width for each $\left[l_{P}, I_{P}\right]$ channel is given by

$$
\Gamma^{\left[l_{P}, I_{P}\right]}=\frac{k^{2 l_{P}+1}}{8 \pi^{2} \Lambda^{2 l_{P}}} \frac{M_{B}}{M_{B}^{*}} \frac{\left|\sum_{q} c_{q}^{\left[l_{P}, I_{P}\right]} \alpha_{q}^{\left[l_{P}, I_{P}\right]} O_{q}^{\left[l_{P}, I_{P}\right]}\right|^{2}}{\left(2 J^{*}+1\right)\left(2 I^{*}+1\right)}
$$

where $k$ is the meson momentum, $\Lambda$ is a scale factor chosen to be $200 \mathrm{MeV}, M_{B}$ is the mass of the ground state baryon resulting from the decay, $M_{B}^{*}$ is the mass of the excited baryon, $J^{*}$ and $I^{*}$ are the spin and isospin of the excited baryon, $c_{q}^{\left[l_{p}, I_{P}\right]}$ are 
dimensionless dynamical coefficients, $O_{q}^{\left[l_{P}, I_{P}\right]}$ are reduced matrix elements of the corresponding operators and $\alpha_{q}^{\left[l_{P}, I_{P}\right]}$ are normalization factors which have been used in the analysis ${ }^{12}$. The mixing of the states occurs within the decay amplitudes.

Table 3: Reduced matrix elements of pion S-wave decays ${ }^{12}$

\begin{tabular}{cccccc}
\hline \hline S-wave decays & $O_{1}^{[0,1]}$ & $O_{2}^{[0,1]}$ & $O_{3}^{[0,1]}$ & $O_{4}^{[0,1]}$ & Overall Factor \\
\hline${ }^{2} N_{1 / 2}^{*} \rightarrow N \pi$ & -1 & $-\frac{1}{2 N_{c}}$ & $-\frac{1}{2 N_{c}}$ & 0 & $\frac{\sqrt{\left(N_{c}+3\right)\left(N_{c}-1\right)}}{3 N_{c}}$ \\
${ }^{2} N_{3 / 2}^{*} \rightarrow \Delta \pi$ & $\frac{1}{3}$ & $-\frac{1}{3 N_{c}}$ & $-\frac{1}{3 N_{c}}$ & $\frac{1}{2 \sqrt{2} N_{c}}$ & $-\frac{\sqrt{3\left(N_{c}+5\right)\left(N_{c}+3\right)}}{\sqrt{6} N_{c}}$ \\
${ }^{4} N_{1 / 2}^{*} \rightarrow N \pi$ & $-\frac{1}{6}$ & $\frac{2}{3 N_{c}}$ & $\frac{1}{6 N_{c}}$ & $\frac{1}{4 \sqrt{2} N_{c}}$ & $-\sqrt{2 \frac{N_{c}-1}{N_{c}}}$ \\
${ }^{4} N_{3 / 2}^{*} \rightarrow \Delta \pi$ & $-\frac{1}{6}$ & $\frac{1}{6 N_{c}}$ & $-\frac{1}{3 N_{c}}$ & 0 & $\sqrt{10 \frac{N_{c}+5}{N_{c}}}$ \\
$\Delta_{1 / 2}^{*} \rightarrow N \pi$ & $\frac{1}{6}$ & $-\frac{1}{6 N_{c}}$ & $-\frac{2}{3 N_{c}}$ & 0 & $-\sqrt{2 \frac{N_{c}-1}{N_{c}}}$ \\
$\Delta_{3 / 2}^{*} \rightarrow \Delta \pi$ & $-\frac{1}{6}$ & $-\frac{1}{3 N_{c}}$ & $\frac{1}{6 N_{c}}$ & $-\frac{1}{4 \sqrt{2} N_{c}}$ & $-\sqrt{10 \frac{N_{c}+5}{N_{c}}}$ \\
\hline$\alpha^{[0,1]}$ & $\frac{3 \sqrt{3}}{2 \sqrt{5}}$ & $\frac{3 \sqrt{3}}{4 \sqrt{5}}$ & $-\frac{3 \sqrt{3}}{4 \sqrt{5}}$ & $\sqrt{\frac{6}{5}}$ & \\
\hline \hline
\end{tabular}

The reduced matrix elements of S-wave decay operators are shown in Table 3. The corresponding dynamical coefficients in the fit are $c s_{i}$ where $i=1,2,3,4$. The reduced matrix elements of the D-wave decay operators are shown in Table 4. The corresponding coefficients for the D-waves in the fit are $c d_{j}$ where $j=1, \ldots, 7$ (since the eighth operator has only a small contribution).

The combined chi-squared value for masses and decays was constructed using Eq.(2). The theoretical expressions for the mixing angles were calculated from the mass matrices constructed from Eq.(5). These expressions were used as input parameters for the mixing of decay amplitudes as given in Eq.(1). 
Table 4: Reduced matrix elements of D-wave decays ${ }^{12}$

\begin{tabular}{|c|c|c|c|c|c|c|c|c|c|}
\hline $\begin{array}{l}\text { D-wave } \\
\text { Decays }\end{array}$ & $O_{1}^{[2,1]}$ & $O_{2}^{[2,1]}$ & $\mathrm{O}_{3}^{[2,1]}$ & $\mathrm{O}_{4}^{[2,1]}$ & $\mathrm{O}_{5}^{[2,1]}$ & $\mathrm{O}_{6}^{[2,1]}$ & $\mathrm{O}_{7}^{[2,1]}$ & $O_{8}^{[2,1]}$ & Overall Factor \\
\hline${ }^{2} N_{1 / 2}^{*} \rightarrow \Delta \pi$ & $-\frac{1}{3 \sqrt{5}}$ & $\frac{1}{3 \sqrt{5} N_{c}}$ & $\frac{1}{3 \sqrt{5} N_{c}}$ & $-\frac{1}{2 \sqrt{10} N_{c}}$ & $-\frac{1}{2 \sqrt{30} N_{c}}$ & $\frac{N_{c}-1}{2 \sqrt{30} N_{c}}$ & $\frac{1}{4 \sqrt{5} N_{c}^{2}}$ & 0 & $5 \frac{\sqrt{\left(N_{c}+3\right)\left(N_{c}+5\right)}}{2 N_{c}}$ \\
\hline${ }^{2} N_{3 / 2}^{*} \rightarrow N \pi$ & $-\frac{1}{\sqrt{5}}$ & $-\frac{1}{2 \sqrt{5} N_{c}}$ & $-\frac{1}{2 \sqrt{5} N_{c}}$ & 0 & 0 & 0 & 0 & 0 & $-5 \frac{\sqrt{\left(N_{c}+3\right)\left(N_{c}-1\right)}}{3 N_{c}}$ \\
\hline${ }^{2} N_{3 / 2}^{*} \rightarrow \Delta \pi$ & $-\frac{1}{3 \sqrt{5}}$ & $\frac{1}{3 \sqrt{5} N_{c}}$ & $\frac{1}{3 \sqrt{5} N_{c}}$ & $-\frac{1}{2 \sqrt{10} N_{c}}$ & $\frac{1}{2 \sqrt{30} N_{c}}$ & $-\frac{N_{c}-1}{2 \sqrt{30} N_{c}}$ & $-\frac{1}{4 \sqrt{5} N_{c}^{2}}$ & 0 & $5 \frac{\sqrt{\left(N_{c}+3\right)\left(N_{c}+5\right)}}{2 N_{c}}$ \\
\hline${ }^{4} N_{1 / 2}^{*} \rightarrow \Delta \pi$ & $-\frac{1}{6 \sqrt{5}}$ & $\frac{1}{6 \sqrt{5} N_{c}}$ & $-\frac{1}{3 \sqrt{5} N_{c}}$ & 0 & $-\frac{1}{\sqrt{30} N_{c}}$ & $\frac{N_{c}-1}{4 \sqrt{30} N_{c}}$ & $-\frac{N_{c}-3}{4 \sqrt{5} N_{c}^{2}}$ & $\frac{\sqrt{7}\left(N_{c}-1\right)}{20 N_{c}^{2}}$ & $-5 \sqrt{\frac{N_{c}+5}{2 N_{c}}}$ \\
\hline${ }^{4} N_{3 / 2}^{*} \rightarrow N \pi$ & $-\frac{1}{4 \sqrt{5}}$ & $\frac{1}{\sqrt{5} N_{c}}$ & $\frac{1}{4 \sqrt{5} N_{c}}$ & $\frac{3}{8 \sqrt{10} N_{c}}$ & $-\frac{3 \sqrt{3}}{8 \sqrt{10} N_{c}}$ & $\frac{3 \sqrt{3}\left(N_{c}+2\right)}{8 \sqrt{10} N_{c}}$ & $-9 \frac{N_{c}+1}{16 \sqrt{5} N_{c}^{2}}$ & 0 & $-\frac{2}{3} \sqrt{5 \frac{N_{c}-1}{N_{c}}}$ \\
\hline${ }^{4} N_{3 / 2}^{*} \rightarrow \Delta \pi$ & $-\frac{1}{3 \sqrt{5}}$ & $\frac{1}{3 \sqrt{5} N_{c}}$ & $-\frac{2}{3 \sqrt{5} N_{c}}$ & 0 & $-\frac{1}{\sqrt{30} N_{c}}$ & $\frac{N_{c}-1}{4 \sqrt{30} N_{c}}$ & $-\frac{N_{c}-3}{4 \sqrt{5} N_{c}^{2}}$ & $-\frac{\sqrt{7}\left(N_{c}-1\right)}{40 N_{c}^{2}}$ & $-2 \sqrt{5 \frac{N_{c}+5}{N_{c}}}$ \\
\hline$N_{5 / 2}^{*} \rightarrow N \pi$ & $-\frac{1}{4 \sqrt{5}}$ & $\frac{1}{\sqrt{5} N_{c}}$ & $\frac{1}{4 \sqrt{5} N_{c}}$ & $\frac{3}{8 \sqrt{10} N_{c}}$ & $\frac{1}{8 \sqrt{30} N_{c}}$ & $-\frac{N_{c}+2}{8 \sqrt{30} N_{c}}$ & $\frac{N_{c}+1}{16 \sqrt{5} N_{c}^{2}}$ & 0 & $-2 \sqrt{5 \frac{N_{c}-1}{N_{c}}}$ \\
\hline$N_{5 / 2}^{*} \rightarrow \Delta \pi$ & $\frac{1}{2 \sqrt{5}}$ & $-\frac{1}{2 \sqrt{5} N_{c}}$ & $\frac{1}{\sqrt{5} N_{c}}$ & 0 & $-\frac{1}{\sqrt{30} N_{c}}$ & $\frac{N_{c}-1}{4 \sqrt{30} N_{c}}$ & $-\frac{N_{c}-3}{4 \sqrt{5} N_{c}^{2}}$ & $-\frac{N_{c}-1}{20 \sqrt{7} N_{c}^{2}}$ & $5 \sqrt{\frac{7}{10} \frac{N_{c}+5}{N_{c}}}$ \\
\hline$\Delta_{1 / 2}^{*} \rightarrow \Delta \pi$ & $\frac{1}{6 \sqrt{5}}$ & $\frac{1}{3 \sqrt{5} N_{c}}$ & $-\frac{1}{6 \sqrt{5} N_{c}}$ & $\frac{1}{4 \sqrt{10} N_{c}}$ & $\frac{1}{4 \sqrt{30} N_{c}}$ & $\frac{N_{c}+2}{20 \sqrt{30} N_{c}}$ & $\frac{3 N_{c}+1}{40 \sqrt{5} N_{c}^{2}}$ & 0 & $5 \sqrt{5 \frac{N_{c}+5}{N_{c}}}$ \\
\hline$\Delta_{3 / 2}^{*} \rightarrow N \pi$ & $-\frac{1}{6 \sqrt{5}}$ & $\frac{1}{6 \sqrt{5} N_{c}}$ & $\frac{2}{3 \sqrt{5} N_{c}}$ & 0 & 0 & 0 & 0 & 0 & $-5 \sqrt{2 \frac{N_{c}-1}{N_{c}}}$ \\
\hline$\Delta_{3 / 2}^{*} \rightarrow \Delta \pi$ & $\frac{1}{6 \sqrt{5}}$ & $\frac{1}{3 \sqrt{5} N_{c}}$ & $-\frac{1}{6 \sqrt{5} N_{c}}$ & $\frac{1}{4 \sqrt{10} N_{c}}$ & $-\frac{1}{4 \sqrt{30} N_{c}}$ & $-\frac{N_{c}+2}{20 \sqrt{30} N_{c}}$ & $-\frac{3 N_{c}+1}{40 \sqrt{5} N_{c}^{2}}$ & 0 & $5 \sqrt{5 \frac{N_{c}+5}{N_{c}}}$ \\
\hline$\alpha^{[2,1]}$ & $\sqrt{\frac{3}{7}}$ & $\frac{3 \sqrt{3}}{10 \sqrt{2}}$ & $\frac{\sqrt{3}}{2 \sqrt{7}}$ & $\frac{2 \sqrt{3}}{5}$ & $\frac{3}{4}$ & $-\frac{12}{5}$ & $-\frac{3 \sqrt{6}}{5}$ & $-\frac{3 \sqrt{3}}{7}$ & \\
\hline
\end{tabular}




\section{RESULTS}

The empirical values for the baryon masses, S-wave and D-wave decays were obtained from the Particle Data Group (PDG) ${ }^{15}$. In Table 5, the results of several fits are presented. It should be noted that the independent fits have been redone using the updated empirical values and do not necessarily reproduce the results of previous studies $^{12,13,14}$. The 3B operators which were omitted from previous analyses ${ }^{12}$ were included in the present analysis since they had a significant contribution to the fits. However, the operator $O_{8}^{[2,1]}$ was not included since its contribution was for only three of the eleven decay channels and would have only resulted in a lesser number of degrees of freedom.

Table 5: Fit Parameters for the Independent and Simultaneous Analyses

\begin{tabular}{|c|c|c|c|c|c|c|c|c|c|c|}
\hline \multicolumn{2}{|c|}{ Coefficients } & \multicolumn{3}{|c|}{ Mass Fit } & \multicolumn{3}{|c|}{ Decay Fit } & \multicolumn{3}{|c|}{$\begin{array}{c}\text { Simultaneous } \\
\text { analysis }\end{array}$} \\
\hline \multirow{6}{*}{ 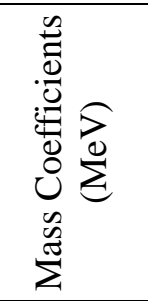 } & $c_{1}$ & 468 & \pm & 1 & & & & 465 & \pm & 1 \\
\hline & $c_{2}$ & -36 & \pm & 7 & & & & -45 & \pm & 7 \\
\hline & $c_{3}$ & 333 & \pm & 45 & & & & 338 & \pm & 52 \\
\hline & $c_{4}$ & 87 & \pm & 22 & & & & 52 & \pm & 19 \\
\hline & $c_{5}$ & 80 & \pm & 12 & & & & 76 & \pm & 12 \\
\hline & $c_{6}$ & 406 & \pm & 6 & & & & 430 & \pm & 6 \\
\hline \multirow{4}{*}{ 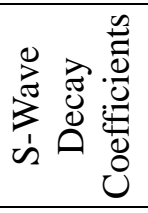 } & $c s_{1}$ & & & & 27.7 & \pm & 2 & 27.9 & \pm & 2 \\
\hline & $c s_{2}$ & & & & 16.6 & \pm & 8 & 23.1 & \pm & 8 \\
\hline & $c s_{3}$ & & & & 20.0 & \pm & 7 & 20.5 & \pm & 8 \\
\hline & $c s_{4}$ & & & & -38.8 & \pm & 7 & -41.1 & \pm & 7 \\
\hline \multirow{7}{*}{ 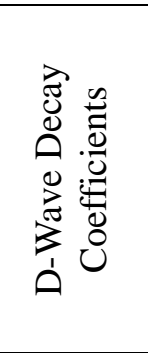 } & $c d_{1}$ & & & & 4.0 & \pm & 0.2 & 4.1 & \pm & 0.2 \\
\hline & $c d_{2}$ & & & & -2.1 & \pm & 1.3 & -2.3 & \pm & 1.3 \\
\hline & $c d_{3}$ & & & & 0.10 & \pm & 2 & 0.42 & \pm & 2 \\
\hline & $c d_{4}$ & & & & 2.2 & \pm & 1.6 & 2.6 & \pm & 1.7 \\
\hline & $c d_{5}$ & & & & -0.3 & \pm & 1.6 & -1.1 & \pm & 1.5 \\
\hline & $c d_{6}$ & & & & -1.8 & \pm & 0.2 & -1.7 & \pm & 0.2 \\
\hline & $c d_{7}$ & & & & -2.0 & \pm & 1.0 & -2.0 & \pm & 1.0 \\
\hline & \multicolumn{3}{|c|}{0.10} & \multicolumn{3}{|c|}{1.99} & \multicolumn{3}{|c|}{2.34} \\
\hline \multicolumn{2}{|l|}{ dof } & \multicolumn{3}{|c|}{1} & \multicolumn{3}{|c|}{1} & \multicolumn{3}{|c|}{3} \\
\hline \multicolumn{2}{|c|}{$\chi_{d o f}^{2}$} & \multicolumn{3}{|c|}{0.10} & \multicolumn{3}{|c|}{1.99} & \multicolumn{3}{|c|}{0.78} \\
\hline \multicolumn{2}{|l|}{$\theta_{1}$} & 0.63 & \pm & 0.08 & 0.40 & 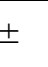 & 0.09 & 0.48 & \pm & 0.09 \\
\hline \multicolumn{2}{|l|}{$\theta_{3}$} & 3.05 & \pm & 0.05 & 2.94 & \pm & 0.07 & 2.97 & \pm & 0.04 \\
\hline
\end{tabular}

The baryon masses and partial widths for strong decays resulting from the different fits are represented graphically in Fig. 1 and Fig. 2. Decay channels for which the values are unknown or unreliable are represented without the empirical values. 


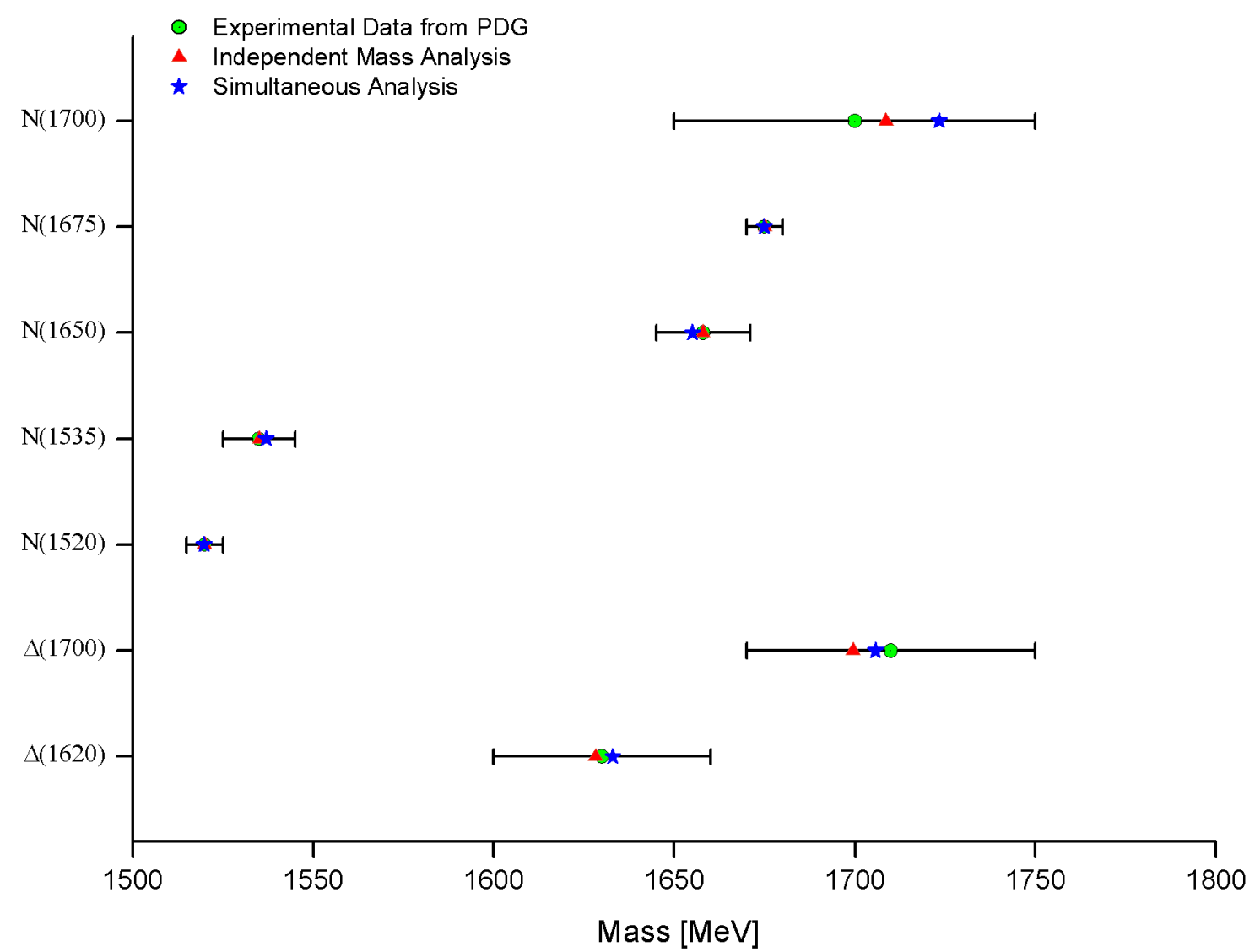

Figure 1: Baryon masses resulting from the fits and the empirical values

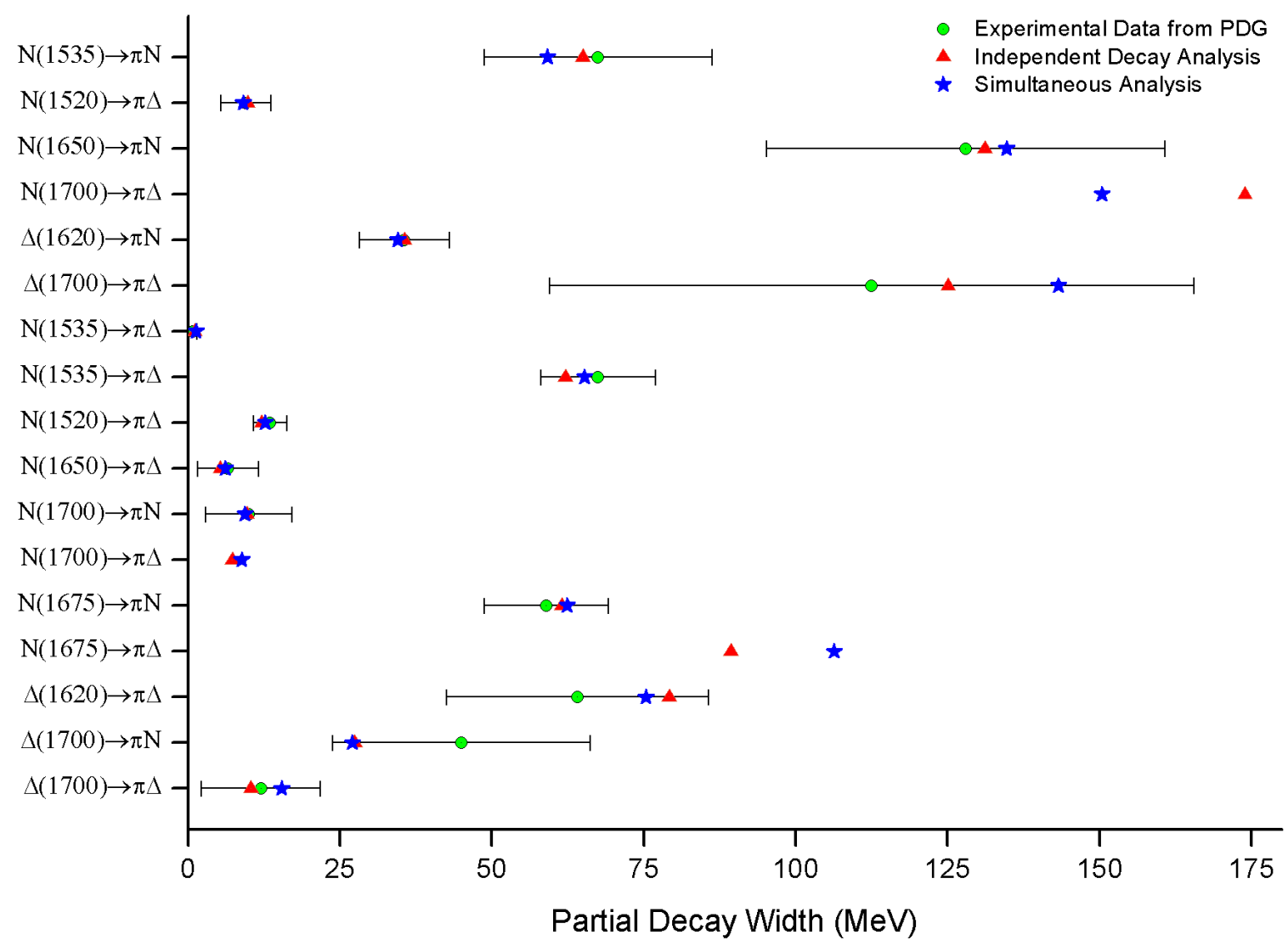

Figure 2: Partial decay widths resulting from the fits and the empirical values 


\section{DISCUSSION AND CONCLUSIONS}

Several noteworthy aspects of the study can be identified. The simultaneous analysis of the masses and strong decays give results consistent with the previous studies $^{12,13,14}$ and give results well within the experimental errors. The experimental errors in the decays are quite high, resulting in higher uncertainty in the subleading order coefficients. The fitted coefficients for S-wave and D-wave decays are separately of natural size which follows from the normalized operators used in the analysis. Hence the relative contributions of the operators can be studied within a particular decay type but not between the S-waves and D-waves. The effective coefficients which carry all the dynamic aspects of the strong interaction, are very much similar in the independent and simultaneous analyses, which confirms the validity of the $1 / N_{c}$ expansion for both masses and decays.

In this study, the mixing angles for the $S=1 / 2,3 / 2$ nucleons are obtained as $\theta_{1}=0.48 \pm 0.09$ and $\theta_{3}=2.97 \pm 0.04$. The value for $\theta_{3}$ is similar to the one obtained in the mass analysis and agrees well with the values in other studies ${ }^{12,13,14}$. The value for $\theta_{1}$ is a significant improvement on the value obtained in the independent decay analysis ${ }^{12}$. The mixing angles obtained were also compared with the results from constituent quark model studies ${ }^{16,17,18,19}$. The obtained values for $\theta_{1}$ and $\theta_{3}$ agree well with the values from mass analyses based on the quark model ${ }^{16,17}$ and have improved upon values from the decay analyses ${ }^{18,19}$.

The most recent study conducted in this sector has been by de Urreta and Scoccola $^{20}$. Their consistent analysis included the study of masses, decays and the helicity amplitudes. Our study did not include the helicity amplitude data due to their unreliability. Furthermore, the extension to $S U(6)$ would not have been possible with the helicity amplitudes due to the scarcity of data. The results for the mixing angles obtained in our study have improved upon the values obtained in the consistent analysis $^{20}$.

The next natural step in this study for the future is the extension of the simultaneous analysis to $S U(6)$. In this sector, several mixing angles should be determined. Also the $S U(6)$ symmetry is moderately broken. Hence, symmetrybreaking operators will also be employed. The $S U(6)$ analysis will however offer a rich source of data unlike observed in the present analysis. It is hoped the success observed in the $S U(4)$ analysis can be reproduced in the $S U(6)$ analysis.

When the results for the complete excited baryon spectrum are available through lattice simulations, application of the $1 / N_{c}$ expansion to the results is expected. This will enable the determination of the quark mass dependence on the dynamical coefficients and hence the nucleon mixing angles. 


\section{REFERENCES}

1. F. Halzen and A.D. Martin, Quarks and Leptons: An Introductory Course in Modern Particle Physics (John Wiley and Sons, Inc., 1984).

2. D. Lichtenberg and S. Rosen (editors), Developments in Quark Theory of Hadrons (Hadronic Press, Nonatum, Mass., 1980) Vol. 1.

3. K. Johnson, The M.I.T. Bag Model, Acta Phys. Polon. B $\underline{6}$, (1975) 865.

4. T.H.R. Skyrme, A Nonlinear Field Theory, Proc. Roy. Soc. Lond. A 260, (1961) 127138.

5. R.F. Dashen, E. Jenkins, and A.V. Manohar, Spin flavor structure of large $N_{c}$ baryons, Phys. Rev. D 1ㅗ, 3697-3727 (1995).

6. G.'t Hooft, A Two-Dimensional Model for Mesons, Nucl. Phys. B $\underline{75}$, (1974) 461.

7. E. Witten, Baryons in the 1/N Expansion, Nucl. Phys. B 160, (1979) 57.

8. J.-L. Gervais and B. Sakita, Large N Baryonic Soliton and Quarks, Phys. Rev. D $\underline{30}$, (1984) 1795.

9. J.-L. Gervais and B. Sakita, Large N QCD Baryon Dynamics : Exact Results from Its Relation to the Static Strong Coupling Theory, Phys. Rev. Lett. 52, (1984) 87.

10. R.F. Dashen and A.V. Manohar, Baryon-pion couplings from large $N_{c} Q C D$, Phys. Lett. B $\underline{315}$, (1993) 425-430.

11. E. Jenkins, Baryon Masses in the $1 / N_{c}$ Expansion, Nucl. Phys. Proc. Suppl. 94 (Bangalore, India, 2001) pp 246-250.

12. J.L. Goity, C. Schat, and N.N. Scoccola, Decays of non-strange negative parity baryons in the 1/N $N_{c}$ expansion, Phys. Rev. D 71, (2005) 034016.

13. C.E. Carlson, C.D. Carone, J.L. Goity, and R.F. Lebed, Masses of orbitally excited baryons in large $N_{c} Q C D$, Phys. Lett. B 438, (1998) 327-335.

14. C.E. Carlson, C.D. Carone, J.L. Goity, and R.F. Lebed, Operator analysis of $l=1$ baryon masses in large $N_{c} Q C D$, Phys. Rev. D $\underline{59}$, (1999) 114008.

15. K. Nakamura, et al. (Particle Data Group), Review of particle physics, J. Phys. G $\underline{37}$, (2010) 075021.

16. N. Isgur and G. Karl, Hyperfine Interactions in Negative Parity Baryons, Phys. Lett. B $\underline{72},(1977) 109$.

17. N. Isgur and G. Karl, P Wave Baryons in the Quark Model, Phys. Rev. D $\underline{18}$, (1978) 4187.

18. R. Koniuk and N. Isgur, Baryon Decays in a Quark Model with Chromodynamics, Phys. Rev. D 21, , (1980) 1868. (Erratum-ibid. D 23 (1981) 818.

19. S. Capstick and W. Roberts, Quark models of baryon masses and decays, Prog. Part. Nucl. Phys. 45, (2000) S241-S331.

20. E.J.G. de Urreta and N.N. Scoccola, Consistent analysis of the [70,1-] baryon properties in the 1/N $N_{c}$ expansion. AIP Conf. Proc. 1343 (Madrid, Spain, 2010) pp 250-252. Consistent analysis of the masses and decays of the [70,1-] baryons in the $1 / N_{c}$ expansion, AIP Conf. Proc. 1432 (Newport News, VA, USA, 2012) pp 265-268. 